\title{
AKT2 expression is associated with glioma malignant progression and required for cell survival and invasion
}

\author{
JUNXIA ZHANG ${ }^{1 *}$, LEI HAN ${ }^{1 *}$, ANLING ZHANG ${ }^{1}$, YINGYI WANG ${ }^{2}$, XIAO YUE $^{2}$, \\ YONGPING YOU ${ }^{2}$, PEIYU PU ${ }^{1}$ and CHUNSHENG KANG ${ }^{1}$ \\ ${ }^{1}$ Department of Neurosurgery, Tianjin Medical University General Hospital and Laboratory of Neuro-Oncology, \\ Tianjin Neurological Institute, Tianjin 300052; ${ }^{2}$ Department of Neurosurgery, The First Affiliated \\ Hospital of Nanjing Medical University, Nanjing 210029, P.R. China
}

Received March 9, 2010; Accepted April 26, 2010

DOI: 10.3892/or_00000829

\begin{abstract}
Recent data suggest that AKT2, one of AKT isoforms, plays an important role in tumorigenesis of human cancers. However, little evidence exists to show the mechanism of AKT2 involved in tumorigenesis. In this study, we show that AKT2 protein expression increased significantly in high grade gliomas in comparison to low grade gliomas and correlated with the expression of NFKB, BCL2, MMP2 and MMP9 by immunostaining. Further, down-regulation of AKT2 expression by antisense AKT2 induced glioma cell apoptosis mediated by NFKB and BCL2. In addition, decreased MMP2 and MMP9 expression in AKT2 knocked-down glioma cells was subsequently detected, consistent with the decreased invasion. These findings indicate that AKT2 expression is associated with more advanced and especially aggressive gliomas and critical for cell survival and invasion.
\end{abstract}

\section{Introduction}

Gliomas, are the most frequent primary tumors that arise in the brain. The most malignant form of glioma, glioblastoma multiforme (Grade IV), is one of the most aggressive human cancers, with a median survival of less than 1 year. Despite recent advances in cancer treatment, this statistic has not changed significantly over the past years. Therefore, it is essential to investigate the mechanism involved in the development and progression of glioma.

Accumulating evidence has demonstrated that PI3K/AKT pathway is one of the important signaling pathways regulating

Correspondence to: Dr Chunsheng Kang, Department of Neurosurgery, Tianjin Medical University General Hospital, Laboratory of Neuro-Oncology, Tianjin Neurological Institute, Tianjin 300052, P.R. China

E-mail: kang97061@yahoo.com

*Contributed equally

Key words: AKT2, glioma, apoptosis, invasion various cellular biological processes, including growth, proliferation, survival, metabolism and motility. AKT, or protein kinase $\mathrm{B}$, a serine/threonine kinase, is composed of three isoforms, AKT1, AKT2 and AKT3. Three isoforms of AKT are structurally homologous and share similar mechanisms of activation, but they also exhibit distinct features and roles (1). AKT1 and AKT2 are widely expressed, whereas AKT3 has restricted expression. Among three isoforms of AKT, AKT2 has been shown to be primarily involved in human cancer and play a central role in tumorigenesis. High expression of AKT2 has been detected in a variety of human cancers, such as laryngeal squamous cell carcinoma, breast cancer and ovarian cancer (2-4). However, there are some reports on the elevated expression of AKT2 in human gliomas $(5,6)$, the relationship between AKT2 expression and glioma grade and the mechanism of AKT2 in gliomagenesis is still not clear.

In the current study, we examined whether AKT2 expression is associated with glioma malignant progression. AKT2 expression increased significantly with the increase in pathologic grade of gliomas and correlated positively with the expression of NFKB, BCL2, MMP2 and MMP9 by immunohistochemistry (IHC). Moreover, reduction of AKT2 by antisense AKT2 inhibited glioma cell survival by induction of apoptosis mediated by NFKB and BCL2. Additionally, down-regulation of AKT2 expression suppressed cell invasion via MMP2 and MMP9.

\section{Materials and methods}

Patients and samples. A glioma tissue microarray was obtained from Shanxi Chaoying Biotechnology (Xi'an, China). Pathologic grades of tumors were defined according to the 2000 WHO criteria as follows: 15 cases with Grade I, 15 cases with Grade II, 15 cases with Grade III, 15 cases with Grade IV. The array dot diameter was $1.5 \mathrm{~mm}$ and each dot represented a tissue spot from one individual specimen that was selected and pathologically confirmed. All microarrays were stored at $4^{\circ} \mathrm{C}$ without light.

Immunohistochemical analysis. Immunostaining was performed on $6 \mu \mathrm{m}$ paraffin sections of tumor specimens by the avidin-biotin-complex (ABC) method as previously 
described (27). Briefly, the sections were incubated with primary antibody (1:100 dilution,) overnight at $4^{\circ} \mathrm{C}$, then incubated with a biotinylated secondary antibody $(1: 200$ dilution) at room temperature for $1 \mathrm{~h}$, followed by the incubation with ABC-peroxidase reagent (1:200 dilution, Vector, USA) for an additional $1 \mathrm{~h}$. After washing with Trisbuffer, the sections were stained with DAB (3,3 diaminobenzidine, $30 \mathrm{mg}$ dissolved in $100 \mathrm{ml}$ Tris-buffer containing $0.03 \% \mathrm{H}_{2} \mathrm{O}_{2}$ ) for $5 \mathrm{~min}$, rinsed in water and counterstained with hematoxylin. The antibodies used in this study were: antibodies to AKT2, NFKB, BCL2, MMP2, MMP9 (Santa Cruz, USA). Negative controls were obtained by substituting primary antibodies with non-immune serum. Sections with no labeling or with $<5 \%$ labeled cells were scored as 0 . Sections were scored as a 1 with labeling of $5-30 \%$ of cells, as a 2 with $31-70 \%$ of cells and as a 3 with labeling of $\geq 71 \%$. The staining intensity was scored similarly, with 0 used for negative staining, 1 for weakly positive, 2 for moderately positive and 3 for strongly positive. The scores for the percentage of positive tumor cells and for the staining intensity were added to generate an immunoreactive score for each specimen. The product of the quantity and intensity scores were calculated such that a final score of $0-1$ indicated negative expression (-), 2-3 indicated weak expression (+), 4-5 indicated moderate expression $(++)$ and 6 indicated strong expression $(+++)$. Each sample was examined separately and scored by two pathologists. Cases with discrepancies in the scores were discussed to reach a consensus.

Cell culture and transfection. Human glioblastoma cell lines (U251, LN229, SNB19) were obtained from the Institute of Biochemistry and Cell Biology, Chinese Academy of Science, Shanghai, China. Human glioblastoma cell line (TJ905) was established in our laboratory (7). The cells were maintained in Dulbecco's modified Eagle's medium (DMEM, Gibco, USA) supplemented with $10 \%$ fetal bovine serum, and incubated at $37^{\circ} \mathrm{C}$ with $5 \% \mathrm{CO}_{2}$. Plasmids pLXSN-AS-AKT2 were kindly provided by Dr Jinquan Cheng (University of South Florida, USA). pLXSN-AS-AKT2 was constructed by subcloning of a 1.2-kb fragment of a human AKT2 cDNA clone into an LXSN retroviral vector in the antisense orientation and the fragment was generated by deleting 70 amino acid residues from the C-terminus of the open reading frame of AKT2. When U251 cells were 70-80\% confluent, pLXSN-AS-AKT2 and empty vector pLXSN were transfected by lipofectamine (Invitrogen, USA). Seventy-two hours after transfection, cells were harvestd for further studies.

Western blot analysis. Parental and transfected cells were washed with pre-chilled phosphate-buffered saline (PBS) three times. The cells were then solubilized in $1 \%$ Nonidet P-40 lysis buffer (20 mM Tris, $\mathrm{pH} 8.0,137 \mathrm{mM} \mathrm{NaCl}, 1 \%$ Nonidet P-40, $10 \%$ glycerol, $1 \mathrm{mM} \mathrm{CaCl}, 1 \mathrm{mM} \mathrm{MgCl} 2,1 \mathrm{mM}$ phenylmethylsulfonyl fluoride, $1 \mathrm{mM}$ sodium fluoride, $1 \mathrm{mM}$ sodium orthovanadate and a protease inhibitor mixture). Total protein lysates ( $40 \mu \mathrm{g} / \mathrm{sample}$ ), were separated by SDSPAGE. The separate proteins were transferred to PVDF membranes. The blot was incubated with primary antibody (AKT2, NFKB, BCL2, MMP2, MMP9), followed by incubation with HRP-conjugated secondary antibody. The specific protein was detected using a super signal protein detection kit (Pierce, USA). After washing with stripping buffer, the PVDF membrane was reprobed with antibody against GAPDH (Santa Cruz, USA).

Cell viability assay. Cells were seeded into 96-well plates at 4000 cells/well. Forty-eight hours after transfection, $20 \mu 1$ of MTT (5 g/l) was added into each well and incubated for $4 \mathrm{~h}$, and then the supernatant was discarded, for 5 consecutive days after treatment. Finally, $200 \mu 1$ of DMSO was added to each well to dissolve the precipitate. Optical density (OD) was measured at the wavelength of $490 \mathrm{~nm}$. The data were presented as the mean $\pm \mathrm{SD}$, derived from triplicate samples of three independent experiments.

Apoptosis assays. Transfected cells were harvestd and then analyzed for the apoptosis ratio using Annexin V FITC Apoptosis Detection kit (Becton-Dickinson, USA) according to the manufacturer's instructions. Annexin V FITC and propidium iodide (PI) double stain was used to evaluate the percentages of apoptosis. Annexin $\mathrm{V}^{-}$and $\mathrm{PI}^{-}$cells were used as controls. Annexin $\mathrm{V}^{+}$and $\mathrm{PI}^{-}$cells were designated as apoptotic and Annexin $\mathrm{V}^{+}$and $\mathrm{PI}^{+}$cells displayed necrotic. Tests were repeated in triplicate.

In vitro transwell invasion assays. Transwell membranes coated with matrigel (Becton-Dickinson) were used to assay invasion of glioma cells in vitro. Transfected cells were plated at $5 \times 10^{4}$ per well in the upper chamber in serum-free medium. FBS $(20 \%)$ was added to the medium in the lower chamber. After incubating for $24 \mathrm{~h}$, non-invading cells were removed from the top well with a cotton swab while the bottom cells were fixed in $95 \%$ ethanol and stained with hematoxylin, and photographed in three independent $\mathrm{x} 400$ fields for each well. Three independent experiments were done and used to calculate fold migration relative to blank control.

In vitro invasive growth assays on matrigel matrix. Transfected cells were seeded into duplicate 24-wells (10000 cells/well) pre-coated with $250 \mathrm{ml}$ matrigel basement membrane matrix (Becton-Dickinson). After incubation for $36 \mathrm{~h}$ photographs were taken at x400 magnification by a light microscope.

Subcutaneous tumor assay. BALB/c-A nude mice at 6 weeks of age were purchased from animal center of the Cancer Institute of Chinese Academy of Medical Science. All experimental procedures were carried out according to the regulations and internal biosafety and bioethics guidelines of Tianjin Medical University and the Tianjin Municipal Science and Technology Commission. U251 glioma subcutaneous model was established as previously described (8). When the subcutaneous tumor reached $50 \mathrm{~mm}^{3}$ in size, the mice were randomly divided into 3 groups (10 subcutaneous tumors/ group). AS-AKT2 group: $10 \mu \mathrm{g}$ pLXSN-AS-AKT2 and $10 \mu \mathrm{l}$ lipofactamine were injected into the xenograft tumor model in a multi-site injection manner. Negative control group: $10 \mu \mathrm{g}$ pLXSN and $10 \mu 1$ lipofactamine were injected into the xenograft tumor model in a multi-site injection manner. Black control group: $10 \mu 1 \mathrm{PBS}$ was injected into the 


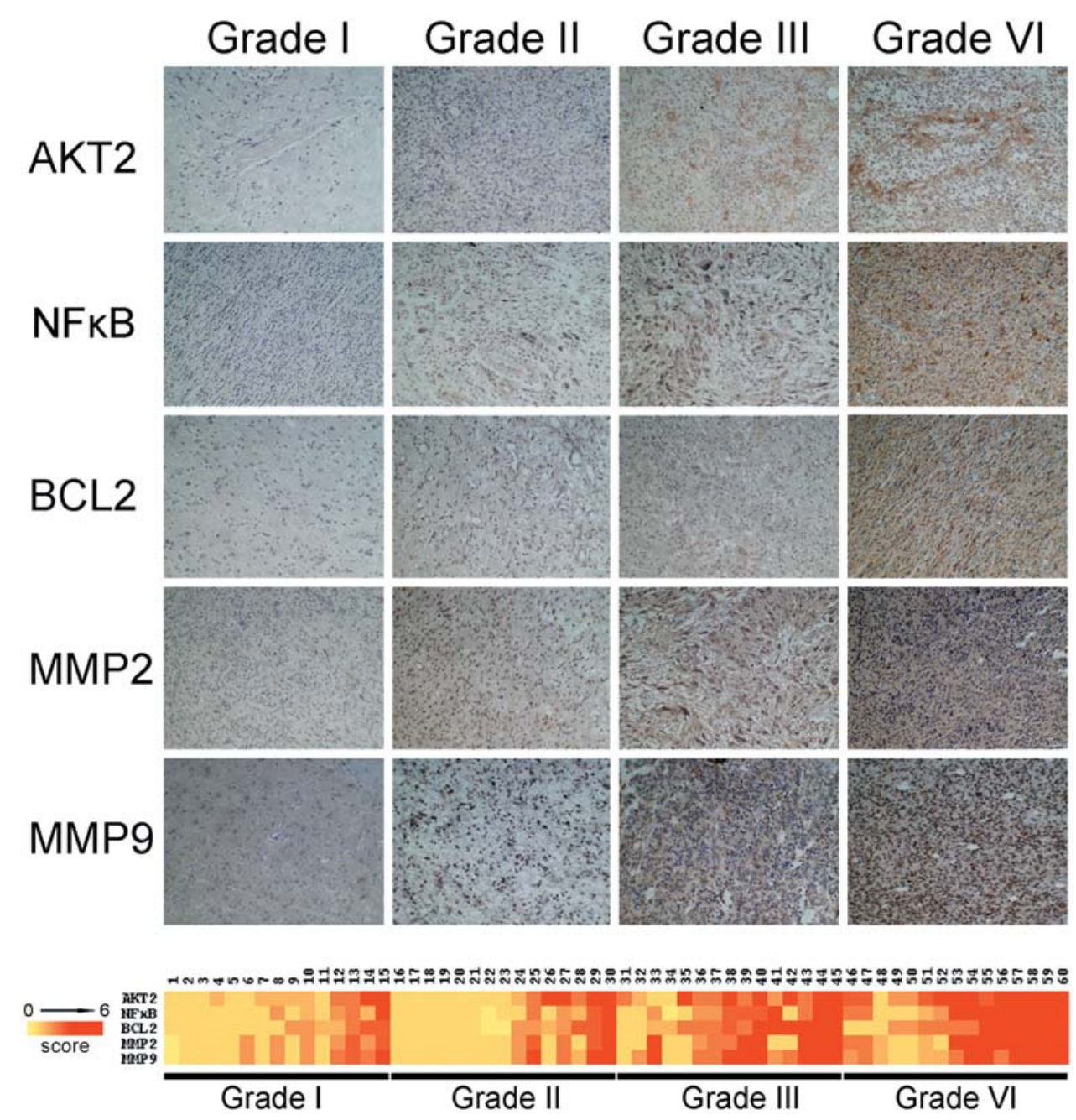

Figure 1. Expression of AKT2, NFאB, BCL2, MMP2 and MMP9 in glioma by IHC. The expression of AKT2, NFkB, BCL2, MMP2 and MMP9 was detected in glioma tissues by IHC. The levels of AKT2, NFKB, BCL2, MMP2 and MMP9 protein increased markedly in high grade gliomas (WHO Grade III and IV) in comparison to low grade gliomas (WHO Grade I and II). Heat map representing gene expression level is determined in a linear scale with a maximum value of 6 immunoreactive score to highlight differences within this range.

xenograft tumor model in a multi-site injection manner. Treatment was conducted at 2-day intervals, twice. The tumor volume was measured with a caliper every 4 days, using the formula volume = length $\mathrm{x}$ width $2 / 2(9)$. At the end of 28-day observation period, the mice bearing xenograft tumors were sacrificed and the tumor tissues were removed for formalin fixation and preparation of paraffin-embedded sections for immunohistochemical analysis.

Statistical analysis. Data were analyzed with SPSS 10.0. ANOVA, t-test, $\chi^{2}$ test and the Pearson's correlation were used to analyze the significance between groups. Statistical significance was assigned to P-values $<0.05$.

\section{Results}

AKT2 expression and its association with pathologic grade in gliomas. Immunostaining analysis revealed AKT2 protein was expressed in gliomas and its total positive rate was $78.3 \%$ (43/60). The levels of AKT2 protein increased markedly in high grade gliomas (WHO Grade III and IV) in comparison to low grade gliomas (WHO Grade I and II). Indeed, 27/30 high grade gliomas exhibited detectable levels of AKT2, while 16/30 low grade gliomas exhibited undetectable levels of the protein $(\mathrm{P}<0.05)$ (Fig. 1). Additionally, there were no significant differences between AKT2 protein expression in the Grade III gliomas and that in the Grade IV gliomas.

In order to explorer the relationship between AKT2 and survival and invasion markers in gliomas, NFKB, BCL2, MMP2 and MMP9 expression was evaluated. NFKB, BCL2, MMP2 and MMP9 expression increased significantly in high grade gliomas compared with low grade gliomas. Representative images of NFKB, BCL2, MMP2 and MMP9 immunostaining are shown in Fig. 1. The Pearson correlation showed that a significant positive correlation existed between AKT2 expression and $\mathrm{NFKB}$ and $\mathrm{BCL} 2$ expression ( $\mathrm{R}=0.7298, \mathrm{P}<0.0001$ and $\mathrm{R}=7857, \mathrm{P}<0.0001$, respectively). A direct correlation between MMP2 and MMP9 expression and AKT2 expression was also observed $(\mathrm{R}=0.6442$, $\mathrm{P}<0.0001$ and $\mathrm{R}=0.6887, \mathrm{P}<0.0001$, respectively) (Fig. 2).

Reduction of AKT2 inhibits glioma cell survival. As shown in Fig. 3A, AKT2 expression in U251 cells was higher than that in other glioma cell lines. Therefore, we chose U251 cells 

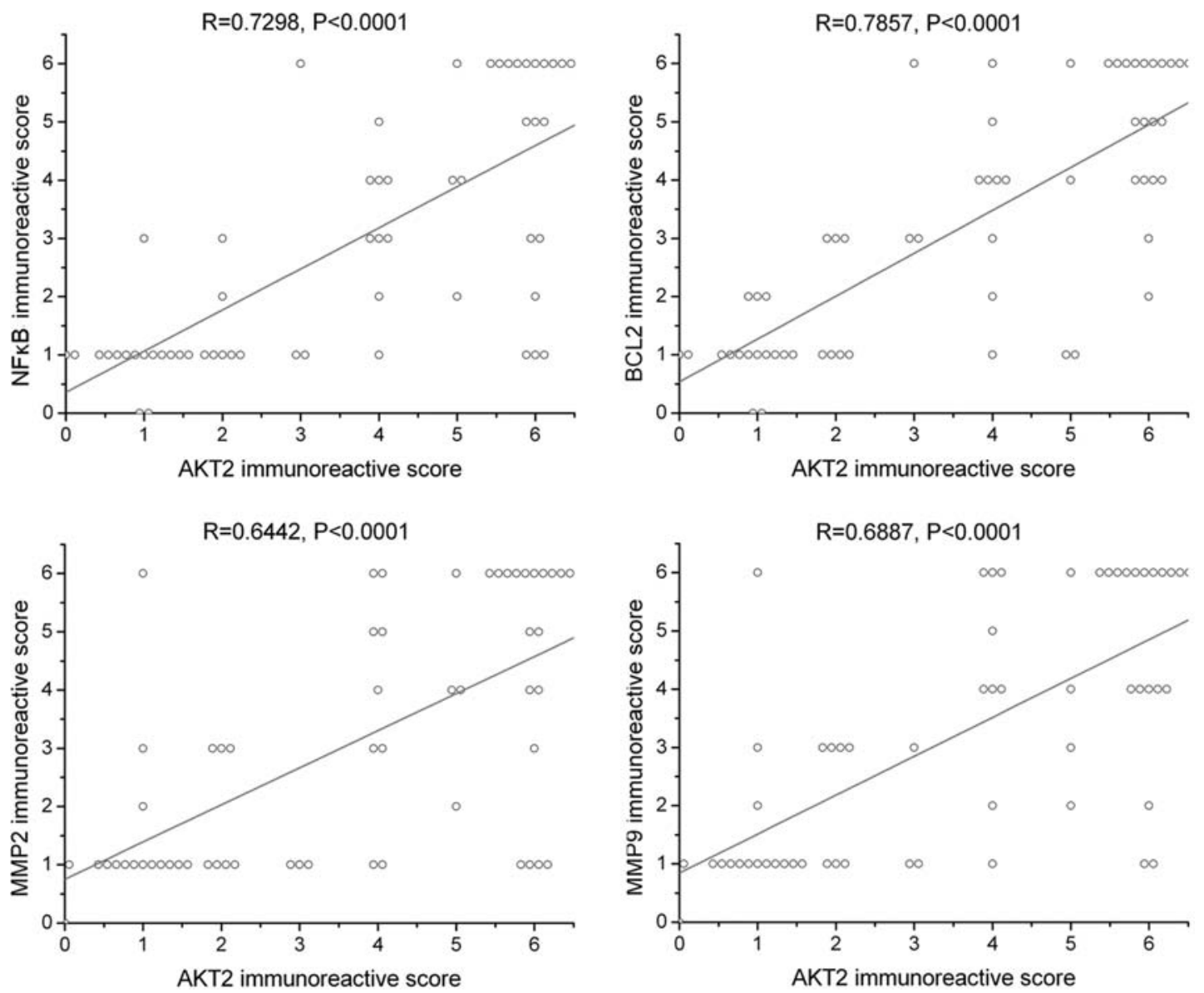

Figure 2. Correlation of AKT2 expression with NFKB, BCL2, MMP2 and MMP9 expression in gliomas. A trend line is provided in each plot, which represents a 'best fit' as determined by simple linear regression. With AKT2 immunoreactive score increase, (A) NFKB, (B) BCL2, (C) MMP2 and (D) MMP9 immunoreactive score increased significantly ( $\mathrm{P}<0.001$ for all, Pearson correlation analysis).

for further studies. In order to efficiently knock down AKT2 expression in glioma cells, antisense AKT2 expression plasmids were transfected into U251 cells. Western blot analysis showed antisense AKT2 induced a marked decrease in the protein levels for the target protein in U251 cells, with minimal effect on the expression of GAPDH, whereas negative plasmid left the AKT2 protein level unchanged (Fig. 3B). These results indicated that antisense AKT2 potently and specifically down-regulated AKT2 expression in glioma cells.

To evaluate the impact of down-regulation of AKT2 expression on glioma cell survival in vitro, MTT assay was employed. Fig. 3C shows that statistically significant cell proliferation inhibition of U251 cells was found in AS-AKT2 group compared with control groups on day 3 . There was no difference between negative control group and blank control group in the whole experiment. Additionally, 3 days after transfection, the apoptosis ratio was measured by Annexin V and PI double stain. Significantly more apoptotic cells were detected in AS-AKT2 group, but no statistical difference of the levels of apoptosis was found in control groups (Fig. 3D). The results indicated that AKT2 appeared to depress glioma cell survival via induction of apoptosis.

Reduction of AKT2 inhibits glioma cell invasion. To investigate whether down-regulation of AKT2 expression could affect invasive ability of glioma cells, in vitro transwell invasion assay was employed. Invasive cells in AS-AKT2 group had the potential to digest the matrigel and migrate through the pores in the membrane. As shown in Fig. 4A, cells in ASAKT2 group showed an average slightly $>50 \%$ defective in migrating through matrigel compared with cells in control groups. Cells with invasive potential exhibit enhanced invasive growth on basement membranes such as matrigel matrix (10). We therefore assayed cell invasive growth on this type of semi-solid surface to measure changes in response to down-regulation of AKT2 expression by antisense AKT2. The majority of cells in control groups was completely adherent $12 \mathrm{~h}$ after seeding, and displayed robust cell growth $24 \mathrm{~h}$ after seeding. While down-regulation of AKT2 expression caused a substantial reduction of cell growth on matrigel in AS-AKT2 group (Fig. 4B). The results suggested that AKT2 is required for glioma cell invasion.

Gene regulation of antisense AKT2 at the protein level in vitro. In order to further explore the molecular mechanism of AKT2 in glioma cell survival and invasion, Western blot analysis was used. NFKB, BCL2, MMP2 and MMP9 are important downsteam molecules of Akt signaling pathway involved in cell survival and invasion. As shown in Fig. 5, an obvious inactivation of $\mathrm{NF \kappa B}$ and BCL2 was observed in U251 after transfection. Consistently, down-regulation of AKT2 

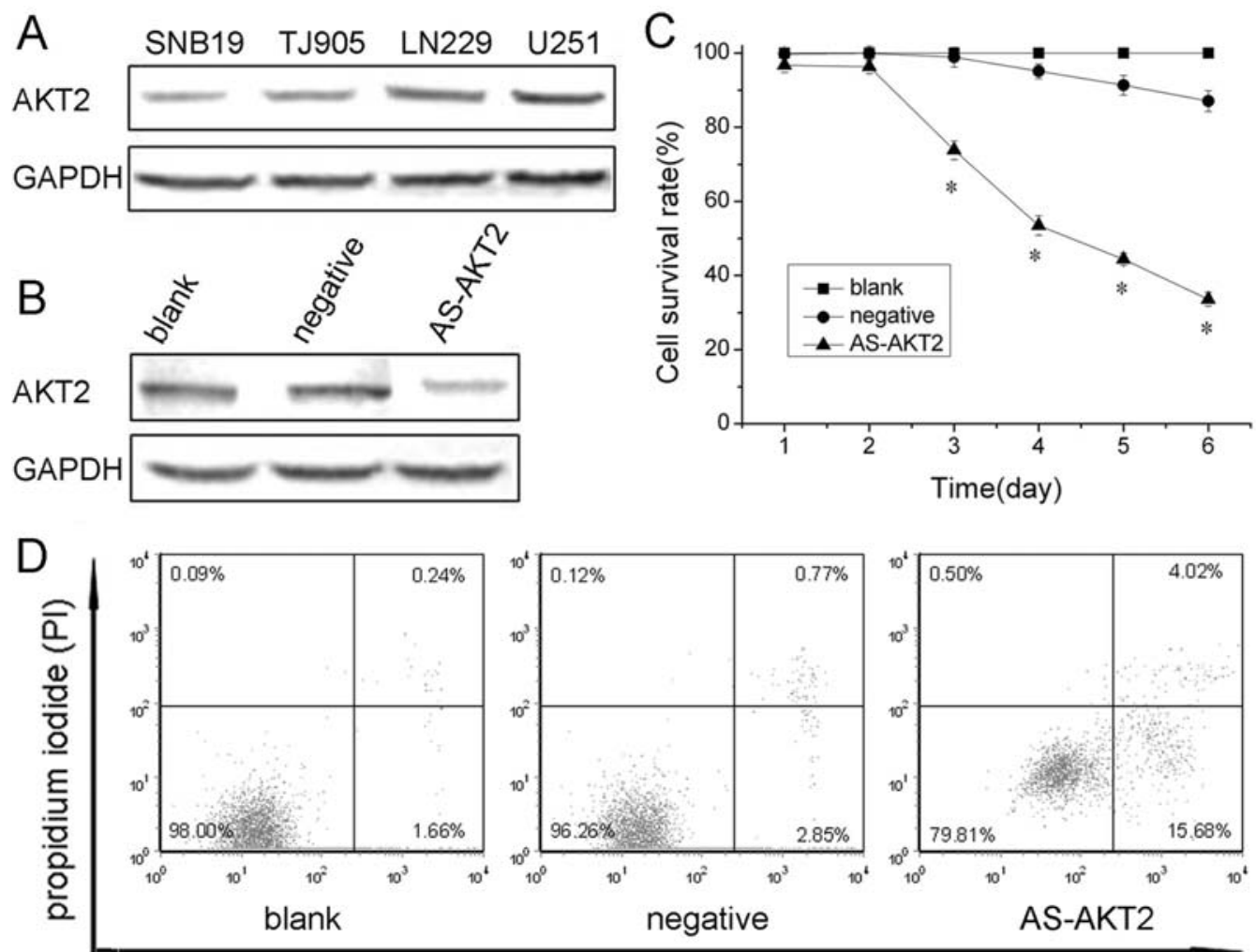

Annexin V

Figure 3. Reduction of AKT2 inhibits glioma cell survival. (A) AKT2 expression in U251 cells was higher than that in other glioma cell lines by Western blotting. (B) Antisense AKT2 induced a marked decrease in AKT2 expression in U251 cells. (C) MTT assay shows that U251 treated with antisense AKT2 proliferated at a significantly lower rate than control groups. There was no difference between blank control group and negative control group in the whole experiment. (D) Reduction of AKT2 suppressed induced cell apoptosis using double stained for Annexin V and propidium iodide (PI). Annexin V'and PI- cells were used as controls. Annexin $\mathrm{V}^{+}$and $\mathrm{PI}-$ cells were designated as apoptotic and Annexin $\mathrm{V}^{+}$and $\mathrm{PI}^{+}$cells were necrotic. ${ }^{*} \mathrm{P}<0.05$ compared with control groups.

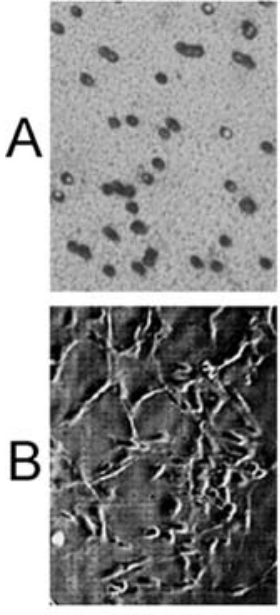

blank
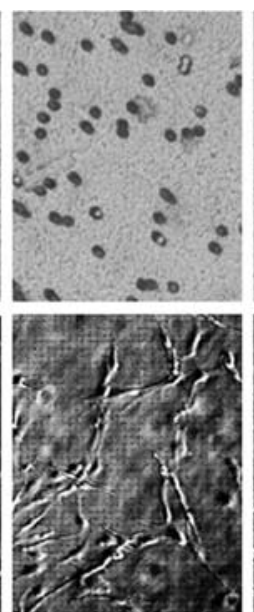

negative
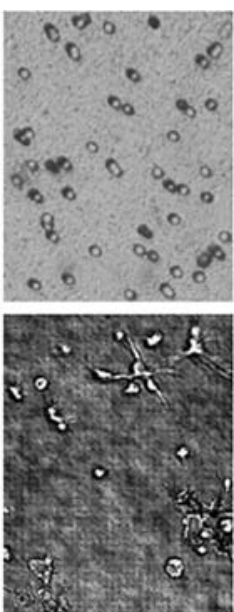

AS-AKT2
Figure 4. Reduction of AKT2 inhibits glioma cell invasion. (A) In vitro transwell invasion assay, penetration proportion of U251 cells was significantly increased in AS-AKT2 group. (B) In vitro invasive growth assays on matrigel matrix, down-regulation of AKT2 expression caused a substantial reduction of cell growth on matrigel in AS-AKT2 group.

expression led to a marked reduction of MMP2 and MMP9 protein expression.

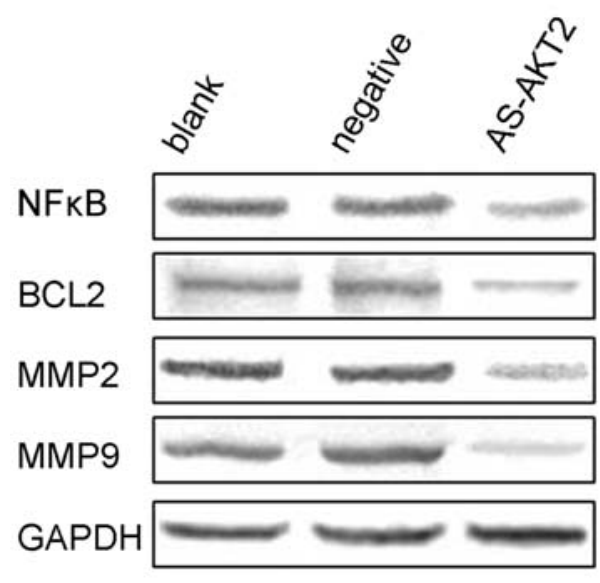

Figure 5. Gene regulation of antisense AKT2 at the protein level in vitro. U251 cells were transfected with antisense AKT2, and the expression of NFKB, BCL2, MMP2 and MMP9 was analyzed by Western blotting. The expression of GAPDH was used as a loading control.

Reduction of AKT2 inhibits glioma growth in vivo. To explore the effect of AKT2 on tumor growth in vivo, we implied a xenograft glioma mouse model. On day 12, the tumors of AS-AKT2 group started to reach statistical significance compared with control groups $(\mathrm{P}<0.05)$. At the termi- 

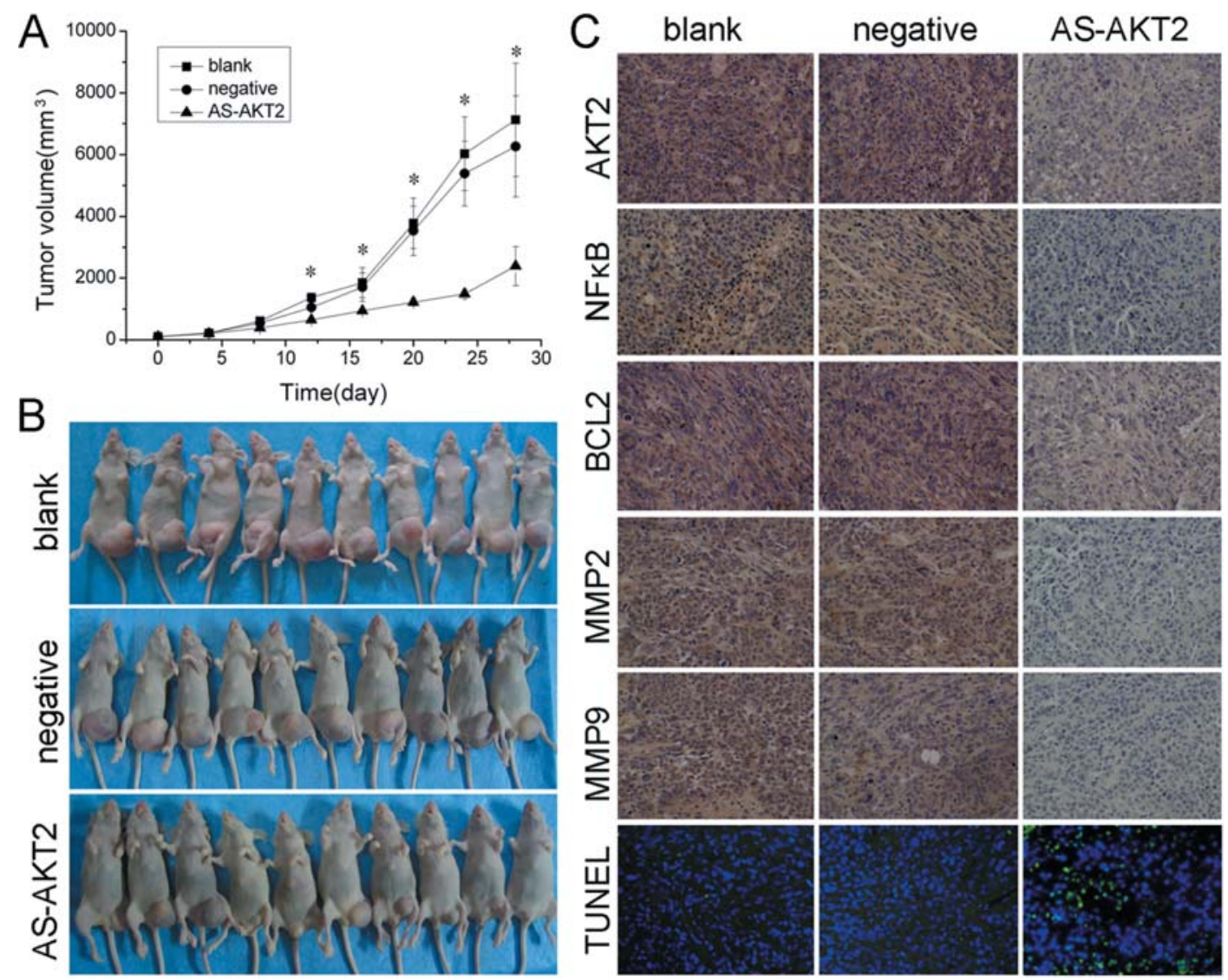

Figure 6. Reduction of AKT2 inhibits glioma growth in vivo. (A) When subcutaneous tumors were established, antisense AKT2 was injected in a multi-site injection manner. Tumor volumes were measured every 4 days during treatment. Down-regulation of AKT2 expression efficiently decreased tumor growth in vivo. (B) A representative animal from 3 groups at the end of the study is shown. (C) Immunohistochemistry analyses of xenograft tumors after antisense AKT2 treatment. The expression level of NFKB, BCL2, MMP2 and MMP9 was suppressed in U251 xenograft tumors of AS-AKT2 group. Additionally, apoptosis cells in subcutaneous U251 tumors treated with antisense AKT2 became obvious by TUNEL staining. ${ }^{*} \mathrm{P}<0.05$ compared with control groups.

nation of the study, the difference in tumor mass between AS-AKT2 group and control groups was marked $(\mathrm{P}<0.01)$. As shown in Fig. 6A and B, the tumor volumes on day 28 were $2392 \pm 633 \mathrm{~mm}^{3}, 6263 \pm 2637 \mathrm{~mm}^{3}$ and $7132 \pm 2835 \mathrm{~mm}^{3}$ in the AS-AKT2 group, negative control group and blank control group, respectively.

After mice were observed for 28 days, the tumor samples were taken, and paraffin-embedded section was prepared for immunohistopathological examination. Similar to the results obtained from the in vitro study, the expression of AKT2, NFKB, BCL2, MMP2 and MMP9 in tumor specimens of ASAKT2 group was prominently down-regulated (Fig. 6C). Cell apoptosis in tumor samples was examined by the TUNEL method, which showed that the number of apoptotic cells was dramatically increased in the tumors from AS-AKT2 treated mice when compared with those in tumors from the control mice.

\section{Discussion}

Cancer cells escape normal growth control mechanisms as a consequence of activating (i.e., gain-of-function) mutations and/or increased expression of one or more cellular protooncogenes and/or inactivating (i.e., loss-of-function) mutations and/or decreased expression of one or more tumor suppressor genes (11). Most oncogene and tumor suppressor gene products are components of signal transduction pathways that control cell cycle entry or exit, promote differentiation, sense DNA damage and initiate repair mechanisms, and/or regulate cell death programs. AKT pathway is the most widely studied component of the pathway networks due to its involvement in cell survival, migration and invasion. In this study, our results revealed that AKT2, one of AKT isoforms, was associated with glioma grade and critical for glioma cell survival and invasion.

Up to date the expression pattern and function of the different AKT isoforms in human tumors are not completely elucidated. It has been shown that AKT1 and AKT2 are ubiquitously expressed in all tissue types so far examined (12), while AKT3 is predominantly expressed in testis and neuronal tissue and is up-regulated in some transformed cells such as in hormone-independent breast and prostate cancer cell lines $(13,14)$. Bellacosa and colleagues found an amplification of AKT2 was associated with undifferentiated carcinomas and poor prognosis (15). Elevated activity levels of AKT2, were associated with high-grade and high-stage ovarian carcinomas (16). Despite high expression of AKT2 in glioma, the relationship between AKT2 expression and tumor grade is still unknown. In our study, AKT2 expression detected by immunohistochemistry and Western blot analysis 
increased significantly with the increase in glioma grade according to the WHO classification. Additionally, AKT2 expression significantly correlated with the antiapoptotic and invasive potential of gliomas, as evaluated by the $\mathrm{NF}_{\mathrm{K}} \mathrm{B}$, BCL2, MMP2 and MMP9 expression. These results suggest that AKT2 expres-sion is associated with more advanced and especially aggres-sive gliomas.

Recent reports have shown that the relative importance of the AKT isoforms in cell survival is cell line-specific (17). In human breast canser ZR-75 cells, AKT1 is the predominant isoform responsible for cell survival. Conversely, in human ovarian carcinoma IGROV1 cells, no single isoform is essential for cell survival. Tumor necrosis factor-like weak inducer of apoptosis (TWEAK), a member of the tumor necrosis factor superfamily, can stimulate glioma cell survival. Depletion of AKT2 expression by siRNA abrogates TWEAKstimulated glioma cell survival, whereas no effect on glioma cell survival is observed after siRNA-mediated depletion of AKT1 expression (18). Our data suggested that down-regulation of AKT2 expression by antisense AKT2 significantly inhibited glioma cell survival through induction of apoptosis mediated by activation of NFKB and BCL2. As far as we know, in a cell-free apoptosis system, mitochondria spontaneously release cytochrome c, leading to apoptotic nuclear morphology. Cytochrome c interacts with Apaf-1, leads to the activation of caspases and finally results in apoptosis. Previous studies have identified that AKT pathway regulates cell apoptosis by inactivation of NFKB and BCL2. NFKB, plays an important role in the regulation of apoptotic cell death via protecting from various apoptotic stimuli in different cellular systems (19). BCL2, which acts to prevent the release of cytochrome $\mathrm{c}$ and caspase activation, is a potent inhibitor of apoptotic cell death (20). These results provide sufficient evidence for the importance of the role of down-regulation of NFKB and BCL2 in antisense AKT2-induced apoptosis.

High levels of MMP2 and MMP9 in tissues are associated with tumor cell invasion (21). MMP2 and MMP9 are thought to be key enzymes involved in the degradation of type IV collagen, which is a component of the extracellular matrix. Additionally, the AKT pathways have been documented to contribute to signal transduction pathways in regulation of MMP2 and MMP9 $(22,23)$. In glioma cells, AKT2 contributes to glioma cell migration and invasion by regulating the formation of cytoskeleton, influencing adhesion and increasing expression of MMP9 (6). In this study, decreased invasive activity was observed in glioma cells by treatment with antisense AKT2. It prompted us to survey its relationship to expression of invasion-associated proteins. Significant difference in decreased MMP2 and MMP9 expression was observed in AS-AKT2 group versus blank control or negative control group both in vitro and in vivo. Taken together, suppression of AKT2 expression can affect suppression of glioma cell invasiveness through MMP2 and MMP9 down-regulation.

In conclusion, the results of the present study indicate that AKT2 expression is associated with glioma malignant progression. AKT2 expression increased significantly with the increase in pathologic grade of gliomas and correlated positively with the expression of NFKB, BCL2, MMP2 and MMP9. Moreover, down-regulation of AKT2 expression by antisense AKT2 inhibited glioma cell survival by induction of apoptosis mediated by NFKB and BCL2. Additionally, down-regulation of AKT2 expression induced invasion suppression via MMP2 and MMP9. Therefore, downregulation of AKT2 expression may offer potential opportunities for glioma gene therapy.

\section{Acknowledgements}

Supported by China National Natural Scientific Found (30971136), Program for New Century Excellent Talents in University (NCET-07-0615) and Natural Science Foundation of Tianjin Municipal Science and Technology Commission (09JZCDJC17600).

\section{References}

1. Woodgett JR: Recent advances in the protein kinase B signaling pathway. Curr Opin Cell Biol 17: 150-157, 2005.

2. Chen Y, He Y, Zhang S, Li L, Zhu X and Liu Y: The expression of oncogene AKT2 in laryngeal squamous cell carcinoma and its clinical significance. Lin Chung Er Bi Yan Hou Tou Jing Wai Ke Za Zhi 23: 539-545, 2009.

3. Morelli C, Lanzino M, Garofalo C, Maris P, Brunelli E, Casaburi I, Catalano S, Bruno R, Sisci D and Ando S: Akt2 inhibition enables the Forkhead transcription factor FoxO3a to a repressive role for estrogen receptor alpha transcriptional activity in breast cancer cells. Mol Cell Biol 30: 857-870, 2010.

4. Weng D, Song X, Xing H, Ma X, Xia X, Weng Y, Zhou J, Xu G, Meng L, Zhu T, Wang S and Ma D: Implication of the Akt2/ survivin pathway as a critical target in paclitaxel treatment in human ovarian cancer cells. Cancer Lett 273: 257-265, 2009.

5. Pu P, Kang C, Li J and Jiang H: Antisense and dominantnegative AKT2 cDNA inhibits glioma cell invasion. Tumour Biol 25: 172-178, 2004.

6. Zhang B, Gu F, She C, Guo H, Li W, Niu R, Fu L, Zhang N and Ma Y: Reduction of Akt2 inhibits migration and invasion of glioma cells. Int J Cancer 125: 585-595, 2009.

7. Wang SL, Li F, Pu PY and Wang GX: The establishment and characteristics of human glioblastoma cell lines TJ899 and TJ905. J Tianjin Med 24: 416-418, 1996.

8. Kang CS, Zhang ZY, Jia ZF, Wang GX, Qiu MZ, Zhou HX, Yu SZ, Chang J, Jiang H and Pu PY: Suppression of EGFR expression by antisense or small interference RNA inhibits U251 glioma cell growth in vitro and in vivo. Cancer Gene Ther 13: 530-538, 2006.

9. You Y, Geng X, Zhao P, Fu Z, Wang C, Chao S, Liu N, Lu A, Gardner K, Pu P, Kong C, Ge Y, Judge SI and Li QQ: Evaluation of combination gene therapy with PTEN and antisense hTERT for malignant glioma in vitro and xenografts. Cell Mol Life Sci 64: 621-631, 2007.

10. Lochter A, Srebrow A, Sympson CJ, Terracio N, Werb Z and Bissell MJ: Misregulation of stromelysin-1 expression in mouse mammary tumor cells accompanies acquisition of stromelysin-1dependent invasive properties. J Biol Chem 272: 5007-5015, 1997.

11. Cantley LC and Neel BG: New insights into tumor suppression: PTEN suppresses tumor formation by restraining the phosphoinositide 3-kinase/AKT pathway. Proc Natl Acad Sci USA 96: 4240-4245, 1999.

12. Meier R, Alessi DR, Cron P, Andjelkovic M and Hemmings BA: Mitogenic activation, phosphorylation, and nuclear translocation of protein kinase Bbeta. J Biol Chem 272: 30491-30497, 1997.

13. Nakatani K, Sakaue H, Thompson DA, Weigel RJ and Roth RA: Identification of a human Akt3 (protein kinase B gamma) which contains the regulatory serine phosphorylation site. Biochem Biophys Res Commun 257: 906-910, 1999.

14. Nakatani K, Thompson DA, Barthel A, Sakaue H, Liu W, Weigel RJ and Roth RA: Up-regulation of Akt3 in estrogen receptor-deficient breast cancers and androgen-independent prostate cancer lines. J Biol Chem 274: 21528-21532, 1999.

15. Bellacosa A, De Feo D, Godwin AK, Bell DW, Cheng JQ, Altomare DA, Wan M, Dubeau L, Scambia G, Masciullo V, Ferrandina G, Benedetti Panici P, Mancuso S, Neri G and Testa JR: Molecular alterations of the AKT2 oncogene in ovarian and breast carcinomas. Int J Cancer 64: 280-285, 1995. 
16. Yuan ZQ, Sun M, Feldman RI, Wang G, Ma X, Jiang C, Coppola D, Nicosia SV and Cheng JQ: Frequent activation of AKT2 and induction of apoptosis by inhibition of phosphoinositide-3-OH kinase/Akt pathway in human ovarian cancer. Oncogene 19: 2324-2330, 2000.

17. Koseoglu S, Lu Z, Kumar C, Kirschmeier P and Zou J: AKT1, AKT2 and AKT3-dependent cell survival is cell line-specific and knockdown of all three isoforms selectively induces apoptosis in 20 human tumor cell lines. Cancer Biol Ther 6 : 755-762, 2007.

18. Fortin SP, Ennis MJ, Savitch BA, Carpentieri D, McDonough WS, Winkles JA, Loftus JC, Kingsley C, Hostetter G and Tran NL: Tumor necrosis factor-like weak inducer of apoptosis stimulation of glioma cell survival is dependent on Akt2 function. Mol Cancer Res 7: 1871-1881, 2009.

19. Shishodia S and Aggarwal BB: Nuclear factor-kappaB activation: a question of life or death. J Biochem Mol Biol 35: 28-40, 2002.
20. Kluck RM, Bossy-Wetzel E, Green DR and Newmeyer DD: The release of cytochrome $\mathrm{c}$ from mitochondria: a primary site for Bcl-2 regulation of apoptosis. Science 275: 1132-1136, 1997.

21. Cawston TE and Wilson AJ: Understanding the role of tissue degrading enzymes and their inhibitors in development and disease. Best Pract Res Clin Rheumatol 20: 983-1002, 2006.

22. Adya R, Tan BK, Punn A, Chen J and Randeva HS: Visfatin induces human endothelial VEGF and MMP-2/9 production via MAPK and PI3K/Akt signalling pathways: novel insights into visfatin-induced angiogenesis. Cardiovasc Res 78: 356-365, 2008.

23. Zhang J, Zhang QY, Fu YC, Wang T, Zhang J, Xu P, Zhou X, $\mathrm{Pu} \mathrm{PY}$ and Kang CS: Expression of p-Akt and COX-2 in gastric adenocarcinomas and adenovirus mediated Akt1 and COX-2 ShRNA suppresses SGC-7901 gastric adenocarcinoma and U251 glioma cell growth in vitro and in vivo. Technol Cancer Res Treat 8: 467-478, 2009. 